\title{
Accurate Modeling of Antennas Using Variable- Fidelity EM Simulations and Co-Kriging
}

\author{
Slawomir Koziel, Stanislav Ogurtsov \\ School of Science and Engineering \\ Reykjavik University \\ Reykjavik, Iceland \\ koziel@ru.is, stanislav@ru.is
}

\author{
Ivo Couckuyt, Tom Dhaene \\ Department of Information Technology \\ Ghent University - IBBT \\ Gent, Belgium \\ ivo.couckuyt@ugent.be,tom.dhaene@ugent.be
}

\begin{abstract}
We present an accurate and low-cost modeling of antenna structures using variable-fidelity electromagnetic (EM) simulations. Our approach exploits sparsely sampled highfidelity (accurate) EM data as well as densely sampled coarsediscretization (low-fidelity) EM simulations that are accommodated into one model using co-kriging technique. By using coarse-discretization simulations, the computational cost of creating the antenna model is greatly reduced compared to conventional approach, where high-fidelity simulations are directly used to set up the model. To our knowledge, this is the first application of co-kriging to antenna modeling. Numerical verification and comparisons with kriging interpolation are given.
\end{abstract}

Keywords-Antenna modeling; electromagnetic (EM) simulation; kriging; co-kriging; computer-aided design (CAD).

\section{INTRODUCTION}

Reliable evaluation of antenna structures can be obtained through electromagnetic (EM) simulation. High-fidelity simulation is CPU intensive, which is a bottleneck for EMbased design tasks such as parametric optimization, statistical analysis, or yield-driven design. Thus, accurate and computationally cheap models of antennas (so-called surrogates) are indispensable.

Cheap antenna models can be obtained using approximation techniques such as polynomial regression [1], radial basis functions [2], kriging [2], [3], support vector regression [4]-[6], artificial neural networks [7]-[10], fuzzy systems [11], or multidimensional Cauchy approximation [12]. However, for good accuracy, these techniques require a large number of training points, particularly if the number of design variables is large.

Here, we consider antenna models constructed using both high- and low-fidelity EM simulations. Simulation of coarsely-discretized antenna structure may not be accurate; however, it is much faster than the high-fidelity one. As we demonstrate, such low-fidelity data can be combined with sparsely sampled high-fidelity simulations using co-kriging [13]. The resulting antenna model is as accurate as the conventional approximation surrogate using much larger number of training data points. The proposed modelling technique is demonstrated using two examples: a ultrawideband planar dipole antenna and a rectangular dielectric resonator antenna. Comparison with conventional kriging interpolation is also given.

\section{ANTENNA MODELING USING CO-KRIGING}

\section{A. Antenna Models}

We consider two types of antenna models. Let $\boldsymbol{R}_{f}(\boldsymbol{x})$ denote an EM-simulated high-fidelity model, which is an accurate representation of the antenna structure. $\boldsymbol{R}_{f}$ is expensive to evaluate (typical simulation time measured in hours). Here, $\boldsymbol{x}$ is a vector of designable (e.g., geometry) parameters. The components of $\boldsymbol{R}_{f}$ may represent, e.g., the antenna reflection coefficient $\left|S_{11}\right|$ over the frequency band of interest. We also consider an auxiliary (low-fidelity) model $\boldsymbol{R}_{c}$ which may be evaluated using the same EM solver, however, with coarser discretization. The low-fidelity model $\boldsymbol{R}_{c}$ is much faster than $\boldsymbol{R}_{f}$ but not as accurate. Therefore, it cannot be normally directly used instead of the high-fidelity model to perform tasks such as design optimization. In this paper, we combine the low- and high-fidelity simulations to create the surrogate model that is almost as accurate as $\boldsymbol{R}_{f}$ but requires much smaller number of high-fidelity training points than the approximation model created using only $\boldsymbol{R}_{f}$ samples.

\section{B. Kriging Interpolation}

Kriging is a popular technique to interpolate deterministic noise-free data [2], [14]. These Gaussian Process based surrogate models are compact and cheap to evaluate. Here, we use kriging as a benchmark technique for comparison with the co-kriging of Section II.C. Let $X_{B . K R}=\left\{\boldsymbol{x}_{K R}{ }^{1}, \boldsymbol{x}_{K R}{ }^{2}, \ldots\right.$, $\left.\boldsymbol{x}_{K R}{ }^{N K R}\right\} \subset X_{R}$ be the base (training) set and $\boldsymbol{R}_{f}\left(X_{B . K R}\right)$ the associated fine model responses. Then, the kriging interpolant, also known as the Best Linear Unbiased Predictor (BLUP), is derived as,

$$
\boldsymbol{R}_{s . K R}(\boldsymbol{x})=M \alpha+r(\boldsymbol{x}) \cdot \Psi^{-1} \cdot\left(\boldsymbol{R}_{f}\left(X_{B . K R}\right)-F \alpha\right)
$$

where $M$ and $F$ are Vandermonde matrices of the test point $\boldsymbol{x}$ and the base set $X_{B . K R}$, respectively. The coefficient vector $\alpha$ is determined by Generalized Least Squares (GLS). $r(\boldsymbol{x})$ is an $1 \times N_{K R}$ vector of correlations between the point $\boldsymbol{x}$ and the base set $X_{B . K R}$, where the entries are $r_{i}(\boldsymbol{x})=\psi\left(\boldsymbol{x}, \boldsymbol{x}_{K R}{ }^{i}\right)$, and $\Psi$ is a 
$N_{K R} \times N_{K R}$ correlation matrix, with the entries given by $\Psi_{i, j}=$ $\psi\left(\boldsymbol{x}_{K R}{ }^{i}, \boldsymbol{x}_{K R}^{j}\right)$. In this work, the exponential correlation function is used, i.e., $\psi(\boldsymbol{x}, \boldsymbol{y})=\exp \left(\sum_{k=1, \ldots, n}-\theta_{k}\left|\boldsymbol{x}^{k}-\boldsymbol{y}^{k}\right|\right)$, where the parameters $\theta_{1}, \ldots, \theta_{n}$ are identified by Maximum Likelihood Estimation (MLE). The regression function is chosen constant, $F=\left[\begin{array}{lll}1 & \ldots & 1\end{array}\right]^{T}$ and $M=(1)$.

\section{Co-Kriging Modeling}

Co-kriging [13] is a type of kriging where the $\boldsymbol{R}_{f}$ and $\boldsymbol{R}_{c}$ model data are combined to enhance the prediction accuracy. Co-kriging is a two-steps process: first a kriging model $\boldsymbol{R}_{S . K R c}$ of the coarse data $\left(X_{B . K R c}, \boldsymbol{R}_{c}\left(X_{B . K R c}\right)\right)$ is constructed and on the residuals of the fine data $\left(X_{B . K R f}, \boldsymbol{R}_{d}\right)$ a second kriging model $\boldsymbol{R}_{\text {s.KRd }}$ is applied, where $\boldsymbol{R}_{d}=\boldsymbol{R}_{f}\left(X_{B . K R f}\right)-\rho \cdot \boldsymbol{R}_{c}\left(X_{B . K R f}\right)$. The parameter $\rho$ is included in the MLE. Note that if the response values $\boldsymbol{R}_{c}\left(X_{B . K R f}\right)$ are not available, they can be approximated by using the first kriging model $\boldsymbol{R}_{s . K R c}$, namely, $\boldsymbol{R}_{c}\left(X_{B . K R f}\right) \approx$ $\boldsymbol{R}_{s . K R c}\left(X_{B . K R f}\right)$. The resulting co-kriging interpolant is defined as

$$
\boldsymbol{R}_{s . C O}(\boldsymbol{x})=M \alpha+r(\boldsymbol{x}) \cdot \Psi^{-1} \cdot\left(\boldsymbol{R}_{d}-F \alpha\right)
$$

where the block matrices $M, F, r(\boldsymbol{x})$ and $\Psi$ can be written in function of the two separate kriging models $\boldsymbol{R}_{\text {S.KRc }}$ and $\boldsymbol{R}_{\text {s.KRd }}$ :

$$
\begin{aligned}
& r(\boldsymbol{x})=\left[\rho \cdot \sigma_{c}^{2} \cdot r_{c}(\boldsymbol{x}), \rho^{2} \cdot \sigma_{c}^{2} \cdot r_{c}\left(\boldsymbol{x}, X_{B . K R_{f}}\right)+\sigma_{d}^{2} \cdot r_{d}(\boldsymbol{x})\right] \\
& \Psi=\left[\begin{array}{cc}
\sigma_{c}^{2} \Psi_{c} & \rho \cdot \sigma_{c}^{2} \cdot \Psi_{c}\left(X_{B . K R_{c}}, X_{B . K R_{f}}\right) \\
0 & \rho^{2} \cdot \sigma_{c}^{2} \cdot \Psi_{c}\left(X_{B . K R_{f}}, X_{B . K R_{f}}\right)+\sigma_{d}^{2} \cdot \Psi_{d}
\end{array}\right] \\
& F=\left[\begin{array}{cc}
F_{c} & 0 \\
\rho \cdot F_{d} & F_{d}
\end{array}\right], \quad M=\left[\begin{array}{ll}
\rho \cdot M_{c} & M_{d}
\end{array}\right]
\end{aligned}
$$

where $\left(F_{c}, \sigma_{c}, \Psi_{c}, M_{c}\right)$ and $\left(F_{d}, \sigma_{d}, \Psi_{d}, M_{d}\right)$ are matrices obtained from the kriging models $\boldsymbol{R}_{S . K R c}$ and $\boldsymbol{R}_{s . K R d}$, respectively (see Section II. $B$ ). In particular, $\sigma_{c}^{2}$ and $\sigma_{d}^{2}$ are process variances, while $\Psi_{c}(\cdot, \cdot)$ and $\Psi_{d}(\cdot, \cdot)$ denote correlation matrices of two datasets with the optimized $\theta_{1}, \ldots, \theta_{n}$ parameters and correlation function of the kriging models $\boldsymbol{R}_{s . K R c}$ and $\boldsymbol{R}_{S . K R d}$, respectively.

\section{VERIFICATION EXAMPLES}

\section{A. UWB Planar Dipole Antenna}

Consider the planar dipole antenna [15] (Fig. 1). The design variables are $\boldsymbol{x}=\left[\begin{array}{llllll}l_{0} & w_{0} & a_{0} & l_{p} & w_{p} & s_{0}\end{array}\right]^{T}$. The high-fidelity model $\boldsymbol{R}_{f}(\sim 10 \mathrm{mln}$ mesh cells, evaluation time 44 minutes $)$ is simulated using the CST MWS transient solver [16]. The lowfidelity model $\boldsymbol{R}_{c}$ is also evaluated in CST $(\sim 100,000$ mesh cells, evaluation time 43 seconds). The antenna models are set up in the region with the center at $\boldsymbol{x}^{0}=\left[\begin{array}{llllll}19 & 13 & 0.5 & 13 & 6 & 1\end{array}\right]^{T}$ and size $\boldsymbol{\delta}=\left[\begin{array}{llllll}1 & 1 & 0.2 & 1 & 1 & 0.2\end{array}\right]^{T}$. The kriging and co-kriging models $\left(\boldsymbol{R}_{\text {S.KR }}, \boldsymbol{R}_{\text {s.CO }}\right)$ are constructed using various numbers of training points (from $N_{K R}=20$ to $N_{K R}=400$ ). Co-kriging models are configured using $400 \boldsymbol{R}_{c}$ samples (the CPU cost of which corresponds to around 6 evaluations of $\boldsymbol{R}_{f}$ ). The quality of the surrogate is assessed using a relative error measure $\| \boldsymbol{R}_{f}(\boldsymbol{x})-$ $\boldsymbol{R}_{s}(\boldsymbol{x})\|/\| \boldsymbol{R}_{f}(\boldsymbol{x}) \|$ expressed in percent. The error is averaged over 50 test designs.

The modeling errors are given in Table I (see also Fig. 2). Note that the co-kriging model accuracy obtained with 20 (50) $\boldsymbol{R}_{f}$ samples is as good as that of the kriging model obtained for 100 (200) samples, which proves that co-kriging and the use of coarse-discretization EM data allows us to greatly reduce the CPU cost of creating accurate antenna model compared to conventional method using solely $\boldsymbol{R}_{f}$ information.

\section{B. Rectangular Dielectric Resonator Antenna}

Consider the rectangular suspended DRA [17] (Fig. 3).

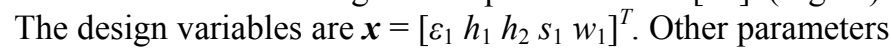
are fixed. The high- and low-fidelity models are evaluated in CST [16] with the following evaluation times: $\boldsymbol{R}_{f} 11$ minutes, and $\boldsymbol{R}_{c} 20 \mathrm{sec}$. The antenna models are set up in the region with the center at $\boldsymbol{x}^{0}=\left[\begin{array}{llllll}10 & 8.5 & 0.5 & 3 & 10\end{array}\right]^{T}$ and size $\boldsymbol{\delta}=\left[\begin{array}{lll}1 & 1 & 0.5\end{array}\right.$ $\left.\begin{array}{ll}1 & 2\end{array}\right]^{T} \mathrm{~mm}$. Similarly as for the previous example, co-kriging allows to substantially reduce the computational cost of creating the accurate antenna model when compared to approximation of the high-fidelity model data only (cf. Table II and Fig. 4).

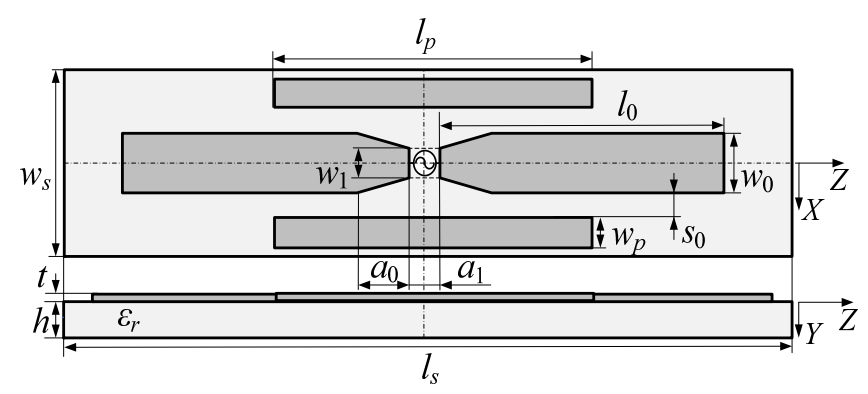

Fig. 1. Dipole antenna geometry [15]: top and side views. The dash-dot lines show the magnetic (YOZ) and the electric (XOY) symmetry walls. The $50 \mathrm{ohm}$ source impedance is not shown at the figure.

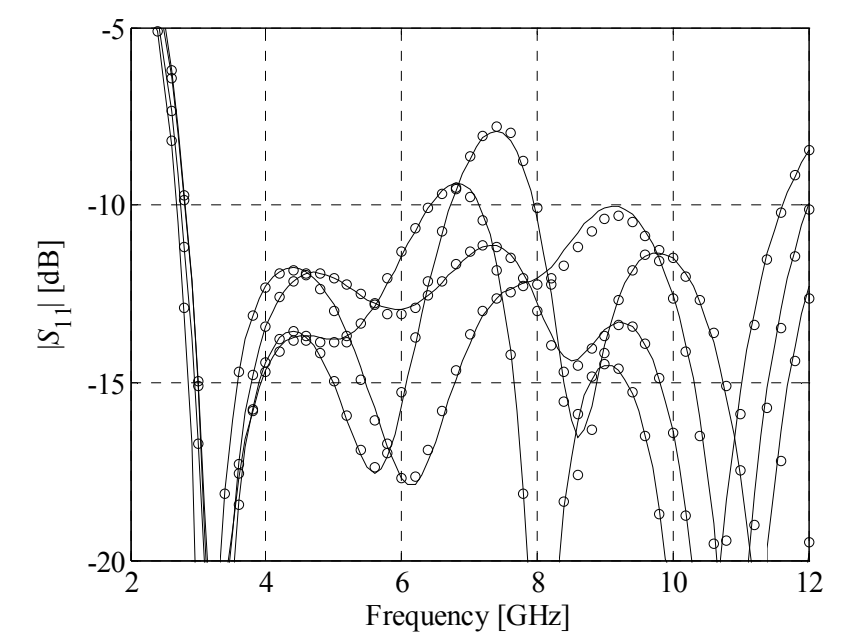

Figure 2. UWB dipole: responses of $\boldsymbol{R}_{f}(-)$ and co-kriging surrogate model (o) at selected test points. Co-kriging model created using 50 evaluations of $\boldsymbol{R}_{f}$ and 400 evaluations of $\boldsymbol{R}_{c}$. 


\section{CONCLUSION}

We presented an antenna modeling methodology using cokriging. We demonstrate that by combining the low- and highfidelity EM simulations, it is possible to create an accurate model of an antenna structure while using limited number of high-fidelity data points.

\section{ACKNOWLEDGMENT}

This work was supported in part by the Icelandic Centre for Research (RANNIS) Grant 110034021. The authors would like to thank Computer Simulation Technology AG, Darmstadt, Germany, for making CST Microwave Studio available.

\section{REFERENCES}

[1] A.I.J. Forrester and A.J. Keane, "Recent advances in surrogate-based optimization," Prog. in Aerospace Sc., vol. 45, no. 1-3, pp. 50-79, 2009.

[2] T.W. Simpson, J. Peplinski, P.N. Koch, and J.K. Allen, "Metamodels for computer-based engineering design: survey and recommendations," Engineering with Computers, vol.17, no. 2, pp. 129-150, July 2001.

[3] J.P.C. Kleijnen, „Kriging metamodeling in simulation: a review.“ European Journal of Operational Research, vol. 192, 707-716, 2009.

[4] A.J. Smola and B. Schölkopf, "A tutorial on support vector regression," Statistics and Computing, vol. 14, no. 3, pp. 199-222, Aug. 2004.

[5] J. Meng and L. Xia, "Support-vector regression model for millimeter wave transition," Int. J. Infrared and Milimeter Waves, vol. 28, no. 5, pp. 413-421, May 2007.

[6] M. Martinez-Ramon and C. Christodoulou, "Support vector machines for antenna array processing and electromagnetics," Synthesis Lectures on Computational Electromagnetics, vol. 1, no. 1, 2006.

[7] H. Kabir, Y. Wang, M. Yu, and Q.J. Zhang, "Neural network inverse modeling and applications to microwave filter design," IEEE Trans. Microwave Theory Tech., vol. 56, no. 4, , pp. 867-879, April 2008.

[8] P. Burrascano, M. Dionigi, C. Fancelli, and M. Mongiardo, "A neural network model for CAD and optimization of microwave filters," IEEE MTT-S Int. Microwave Symp. Dig., Baltimore, MD, 1998, pp. 13-16.

[9] L. Zhang, J. Xu, M.C.E. Yagoub, R. Ding, and Q.-J. Zhang, "Efficient analytical formulation and sensitivity analysis of neuro-space mapping for nonlinear microwave device modeling," IEEE Trans. Microwave Theory Tech., vol. 53, no. 9, pp. 2752-2767, Sept. 2005.

[10] L. Zhang, J.J. Xu, M. Yagoub, R.T. Ding, and Q.J. Zhang, "Neuro-space mapping technique for nonlinear device modeling and large signal simulation," IEEE MTT-S Int. Microwave Symp. Dig., Philadelphia, PA, June 2003, pp. 173-176.

[11] V. Miraftab and R.R. Mansour, "Computer-aided tuning of microwave filters using fuzzy logic," IEEE Trans. Microwave Theory Tech., vol. 50, no. 12, pp. 2781-2788, Dec. 2002.

[12] G.S.A. Shaker, M.H. Bakr, N. Sangary, and S. Safavi-Naeini, "Accelerated antenna design methodology exploiting parameterized Cauchy models," PIER-99, pp. 279-309, 2009.

[13] M.C. Kennedy and A. O'Hagan, "Predicting the output from complex computer code when fast approximations are available", Biometrika, vol. 87 , pp. 1-13, 2000.

[14] D. Gorissen, K. Crombecq, I. Couckuyt, P. Demeester, and T. Dhaene, "A surrogate modeling and adaptive sampling toolbox for computer based design," J. Machine Learning Research, vol. 11, pp. 2051-2055, 2010.

[15] T. G. Spence and D. H. Werner, "A novel miniature broadband/multiband antenna based on an end-loaded planar open-sleeve dipole," IEEE Trans. Antennas Propag., vol. 54, no. 12, pp. 3614-3620, Dec. 2006.
[16] CST Microwave Studio, ver. 2011, CST AG, Bad Nauheimer Str. 19, D64289 Darmstadt, Germany, 2011.

[17] A. Petosa, Dielectric resonator antenna handbook, Artech House, 2007.

TABLE I. UWB Dipole ANTENNA: MODELING RESUlts

\begin{tabular}{|c|c|c|c|c|c|}
\hline \multirow{2}{*}{ Model } & \multicolumn{5}{|c|}{ Average Modeling Error [\%] } \\
\cline { 2 - 6 } & $N_{K R}=20$ & $N_{K R}=50$ & $N_{K R}=100$ & $N_{K R}=200$ & $N_{K R}=400$ \\
\hline $\boldsymbol{R}_{S . K R}$ & 17.5 & 5.6 & 4.3 & 2.8 & 2.0 \\
\hline $\boldsymbol{R}_{S . C O}$ & 4.2 & 2.6 & 2.4 & 2.0 & 1.9 \\
\hline
\end{tabular}

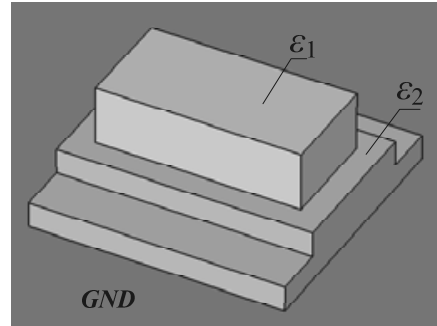

(a)

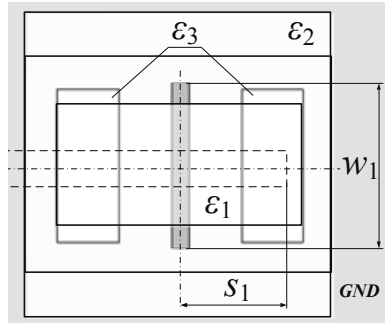

(b)

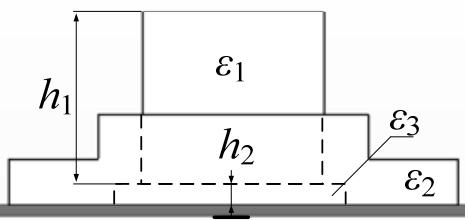

(c)

Fig. 1. DRA geometry [17]: (a) 3D view, (b) top view, and (c) side view.

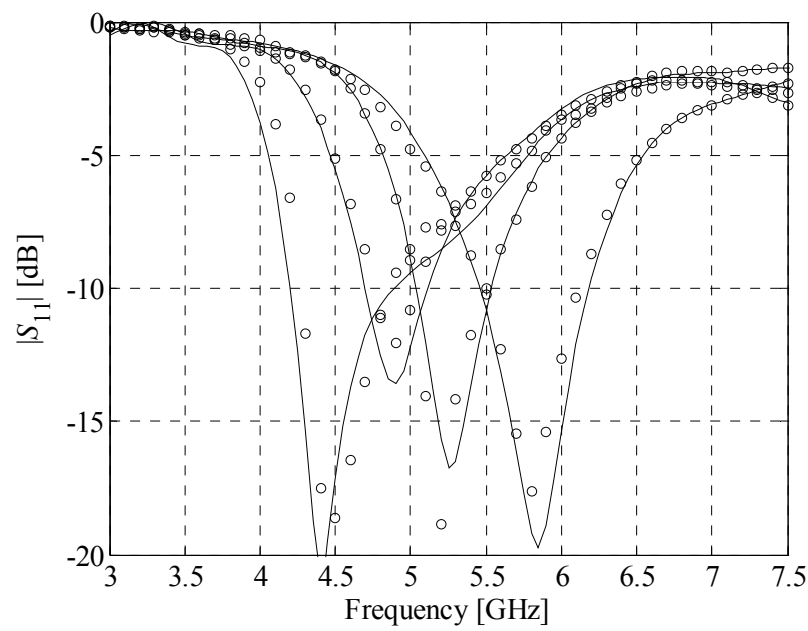

Figure 2. Rectangular DRA: responses of $\boldsymbol{R}_{f}(-)$ and co-kriging surrogate (o) at selected test points. Co-kriging model created using 50 evaluations of $\boldsymbol{R}_{f}$ and 400 evaluations of $\boldsymbol{R}_{c}$.

TABLE II. RECTANGULAR DRA: MODELING RESUlTS

\begin{tabular}{|c|c|c|c|c|c|}
\hline \multirow{2}{*}{ Model } & \multicolumn{5}{|c|}{ Average Modeling Error [\%] } \\
\cline { 2 - 6 } & $N_{K R}=20$ & $N_{K R}=50$ & $N_{K R}=100$ & $N_{K R}=200$ & $N_{K R}=400$ \\
\hline $\boldsymbol{R}_{\text {s.KR }}$ & 12.1 & 8.8 & 6.9 & 5.2 & 3.6 \\
\hline $\boldsymbol{R}_{\text {s. } C O}$ & 6.7 & 5.4 & 5.0 & 4.1 & 3.5 \\
\hline
\end{tabular}

